\title{
Acceptability of Self-Sampling HPV Testing Among Thai Women for Cervical Cancer Screening
}

\author{
Shina Oranratanaphan*, Wichai Termrungruanglert, Nipon Khemapech
}

\begin{abstract}
Background: Acceptability of self-sampling HPV testing is confirmed worldwide. However, some cultural differences may affect this question. Therefore, this study was conducted to evaluate the acceptability of selfsampling HPV testing in Thai women. Materials and Methods: One hundred women aged 30-65 years with an intact cervix were included in this study. The participants were asked to do the Pap test by physicians and then brush type self-sampling instruments were assigned for self-collection and finally completed a questionnaire for acceptability evaluation. The questionnaire contains 2 parts. Part one covered general information of the participants and part two is the acceptability questions. Results: Mean age was $\mathbf{4 0 . 6}$ years. The incidence of high risk HPV detection in this study was $16 \%$. The most common reason for doing Pap smear was for annual checkup. On the topic of ease of use, $85 \%$ of the subjects agreed. Most of the participants $(82 \%)$ reported that they felt less pain. However, reliability of the result was not satisfactory because $37 \%$ of the participants hesitated to rely on the results of the test. According to the price, if the price is less than 1,000 Baht (32.59 Baht $=1 \mathrm{USD})$, $82 \%$ of the subjects would use it for their next screening. Conclusions: The acceptability of self-sampling device in this study is quite good but the reliability of the test was questioned by some of the participants. Moreover, the price of the test in Thailand may also influence the acceptability of the test.
\end{abstract}

Keywords: Self sampling - HPV testing - acceptability - secondary prevention - pap smear - cervical cancer

Asian Pac J Cancer Prev, 15 (17), 7437-7441

\section{Introduction}

Cervical cancer is the second most common female cancer in Thailand and worldwide (IARC, 2005; Ferlay et al., 2010). Persistence high risk HPV infection is known as a leading cause of cervical cancer (Schiffman and Kjaer, 2003; IARC, 2005). Most of the cervical cancer cases developed in the developing countries. Despite of the wide spreading of the disease, cervical cancer is one of the preventable cancers. Pap smear either conventional or liquid based methods have also been proved that can be used to decrease the incidence of cervical cancer. Pap smear helps physicians to detect precancerous lesions and treat before the lesions progress to cancer. Moreover, several methods have been used to detect precancerous lesions of the cervix such as VIA (visual inspection with acetic acid) and HPV DNA testing either physician taking or self-sampling method (WHO, 2006). These screening tests can reduced the incidence of cervical cancer nearly $80 \%$ in developed countries (WHO, 2006). Screening for cervical cancer by using HPV DNA testing can reduce the burden of HPV mediated carcinogenesis especially cervical cancer (Saumya et al., 2012). HPV DNA testing has been proven an effective and better method for cervical cancer screening than cytology (Li et al., 2014). On the other hand, HPV DNA testing has a high sensitivity. Too high sensitivity of the test may lead to unnecessary colposcopy. Using the combination of HPV DNA testing and cytology can reduce referral rate to colposcopy without increasing false negative rate (Mehmet et al., 2013). However, the incidence of HPV infection is varied by age, ethnic, cultural and genetic predisposition (Saumya et al., 2012).

Although there are so many screening tools that have been proven to decrease cervical cancer incidence, the incidence of cervical cancer in Thailand is still high. The incidence of cervical cancer in Thailand increased from 6,954 new cases in 1999 to 9,999 new cases in the year 2008 (MOPH, 2008; Ferlay et al., 2010). From the recent database of cancer surveillance in Thailand, the incidence of cervical cancer in Thailand was 9,999 cases per year and mortality rate was 5,216 cases per year (Ferlay et al., 2010). Moreover, most of the cervical cancer in Thailand is in advanced stage. The reason for increment of the incidence is the poor screening coverage (Sriamporn et al., 2006). There was a report about the coverage of Pap smear in Thai women. The result found that only $25-38 \%$ of Thai female aged 30-65 years have ever been screen 
at least once in their life time and the rest of them have never been screen. On the other hand, the screening rate in developed country is $70 \%$ (Bundhamcharoen et al., 2002; MOPH 2007). Because of the national policy before 2004 (Ngan et al., 2011), there has not had the national policy for regular annual checkup for all women. The recent Thai national policy is screening for every 5 years. Therefore, many people have to pay by themselves for annual Pap test. Furthermore, the important obstacle of Pap test is embarrassment. There was a survey in Thailand for the reasons that women avoid going to do the Pap test. The most significant reason is the embarrassment (Thurston and Scott., 2005; Oranratanaphan et al., 2010). The other reasons are fear of pain from vaginal speculum examination, too busy to go and do not have any symptoms (Oranratanaphan et al., 2010). The similar reasons for avoid doing Pap smear is reported in other developing countries (Mustafa et al., 2014).

Self-sampling HPV test has been proved worldwide about the accuracy for HPV detection (Forrest et al., 2004; Stervall et al., 2007). It also has been proved that it has high concordance rate between physician taken specimen and self-sampling in HPV detection rate (Szarewski et al., 2007; Dijkstra et al., 2012; van Baars et al., 2012; Snijder et al., 2013). Moreover, it also has been proved that it can increase coverage for cervical cancer screening in the national screening program in some countries. There are some studies showed that the response rate of selfsampling HPV testing is better than notification letters in women who did not attend annual screening program (non-attendee) (Bais et al., 2007; Virtanen et al., 2011; Lindell et al., 2012; Tamalet et al., 2013). Acceptability of self-sampling HPV testing is also proved worldwide. Women from most countries accept that Self sampling HPV testing is easy to use, convenience, less pain and less embarrassment (Dzuba et al., 2002; Anhang et al., 2005; Stenvall et al., 2007; Arriba et al., 2010). However, there are some groups of people such as Muslim people that do not accept self-sampling HPV testing (Szarewski et al., 2009). The reason for unaccepted is based on cultural and behavioral differences. Some cultures, believes and behavior of Thai women are also difference from Western country. So, the acceptability of self-sampling HPV testing in Thai women may not correspond with the results from the western countries.

For that reason, this study is conducted to evaluate the acceptability of self-sampling HPV testing in Thai women. The result of this study may be basic information in generalize the utility of self-sampling HPV testing in general population in Thailand.

\section{Materials and Methods}

After the approval from the Ethical Committee was accepted, the study was conducted. Sample size was calculated. One Hundred women have to be included to this study. The participants were recruited from women attending for cervical cancer screening at the outpatient clinic of King Chulalongkorn Memorial Hospital. Women aged 30-65 years with intact cervix were included. The exclusion criteria were women who previously hysterectomies or previously diagnosed as cervical cancer or precancerous lesions. The participants who cannot read or write were also excluded. The women meeting the inclusion criteria and not having exclusion criteria were informed about this study. All the participants have to sign inform consent voluntarily before enroll to the study.

Data collection was performed from January 2014 to April 2014. All the participants were assigned to do the Pap test by physicians and after that brush type self-sampling HPV testing instruments were assigned to participants to collect the specimens by themselves. The instruction of self-sampling HPV test was also handed to them as a leaflet in the package of Self-sampling kit. After complete both procedures, the participants had to answer the questions in the questionnaire by themselves and handed back to the investigator after complete all steps. The questionnaires were collected separately from the Pap and self-sampling specimens. The identification data were labelled only on the specimen for the treatment and follow up reason. In cases that cervical cancer screening result was abnormal, identification data are used to call the patients back for further investigation and treatment. However, there are no identification data of the participant on the questionnaire. Therefore, the answer of the participants is still confidential.

The questionnaire contains 2 parts. Part one is general information of the participants such as age, occupation, salary, marital status and parity. Part two is the acceptability questions to assess the acceptability of self-sampling HPV testing. The questions used Likert's scales for scoring each aspect of the questions. The scales range from 1 to 5 . The meaning of the score number is written at the heading of the questionnaire. Score 1 means strongly disagree. Score 2 means disagree. Score 3 is equivalence. Score 4 is agree and Score 5 means strongly agree. The score 4 and 5 are classified as satisfaction or acceptance. Score 3 or less are verified as poor acceptability. The question is about easily to use, embarrassment, pain, reliability of the results, privacy and cost of the instrument.

After all questionnaires were collected, the data was analyzed by SPSS version 16.0. The data is analyzed in mean mode median and percentage. Presentation of the analyzed data is shown in tables. After finish the study, the Pap smear and HPV testing results will be sent to the participants. The participants who have abnormal results of either from Pap smear or HPV testing will call back for further investigation and treatment.

\section{Results}

After complete data collection from all participants, the entire questionnaires were evaluated. The data were processed with SPSS version 16.0. All the information were analyzed in mean, mode, median and percentage. Mean age of the participants was 40.6 years (SD 9.3). The details of general characteristics were displayed in Table 1. Their occupation was varies from Government employee to housewife or unemployed. Housewife in this study means a woman who does the housework in their own house without any officially income. However, the majority of the participants were employee either 
temporary or full time employee. The majority of the participants in this study earned 10,000-30,000 Baht per month (32.59 Baht = 1 USD) which was correlated to the average income of Thai population claimed by IMF 2012. From the study, $83 \%$ of the subjects earn 10,000-30,000 Baht per month, $15 \%$ earn less than 10000 Baht per month and only $2 \%$ earn 30,001-50,000 Baht per month. The general information data of the participants were shown in table 1 . Most of the participants were married. From the aspect of parity, $22 \%$ did not have any child, while $78 \%$ of the participants have at least one child. Half of the participants have delivered vaginally $(52.6 \%)$. The rest of them have delivered by Cesarean section. While, Most of them (81\%) have performed Pap smear at least once in their lifetime, $19 \%$ of them have never performed Pap smear. According to the duration from previous Pap smear, 19\% never performed Pap smear. Only $49 \%$ of the participants performed Pap smear in last year, $12 \%$

Table 1. General Characteristics of the Participants

\begin{tabular}{|c|c|c|}
\hline \multicolumn{2}{|l|}{ Character } & \multirow{2}{*}{$\frac{\text { Percent }}{7}$} \\
\hline Occupation & Government employee & \\
\hline & Employee & 27 \\
\hline & Temporary employee & 38 \\
\hline & Freelance & 18 \\
\hline & Housewife and unemployed & 10 \\
\hline \multirow[t]{3}{*}{ Income per month } & $<10000$ & 15 \\
\hline & at $10001-30000$ & 83 \\
\hline & at $30001-50000$ & 2 \\
\hline \multirow[t]{4}{*}{ Marital status } & Single & 18 \\
\hline & Marry & 79 \\
\hline & Widow & 2 \\
\hline & Divorce & 1 \\
\hline \multirow[t]{4}{*}{ Parity } & 0 & 22 \\
\hline & 1 & 38 \\
\hline & 2 & 28 \\
\hline & 3 or more & 12 \\
\hline \multirow[t]{3}{*}{ Delivery method } & Non delivery & 22 \\
\hline & Vaginal delivery & 41 \\
\hline & Cesarean section & 37 \\
\hline \multicolumn{3}{|c|}{ Have ever done pap smear } \\
\hline & Yes & 81 \\
\hline & No & 19 \\
\hline \multicolumn{3}{|c|}{ Duration form Last Pap (yr) } \\
\hline & no previous Pap & 19 \\
\hline & 11 & 49 \\
\hline & 2 & 12 \\
\hline & 3 or more & 20 \\
\hline \multicolumn{3}{|c|}{ Reason for current Pap test } \\
\hline & Annual check up & 66 \\
\hline & Abnormal symptoms & 15 \\
\hline & Cancer in relation & 10 \\
\hline & Postpartum & 9 \\
\hline
\end{tabular}

Table 2. Acceptability for Self Sampling HPV Testing

\begin{tabular}{lccc}
\hline Factors & Score 3 or less & Score 4 & Score 5 \\
\hline Easily to use & 15 & 38 & 47 \\
Less pain & 18 & 41 & 41 \\
Reliable result & 37 & 51 & 12 \\
Comfortable & 11 & 37 & 52 \\
Less embarrassment & 20 & 30 & 50 \\
Prefer to do it at home & 17 & 35 & 48 \\
Suggest the other to use & 10 & 53 & 37 \\
Will use this method next time & 21 & 40 & 39 \\
\hline
\end{tabular}
performed Pap test last 2 years and 20\% performed Pap smear more than 3 years ago. The most common reason for doing Pap test is for annual checkup which was $65 \%$ of the participants. The second most common reason is having abnormal symptoms such as leucorrhea or pelvic pain. The rest for the participants come for Pap smear because they have some history of cancer in their family or for postpartum checkup.

For the acceptability part, Likert scale was used to evaluate the satisfaction and acceptability of each topic. The scales range from 1 to 5 . Score 1 means strongly disagree. Score 2 means disagree. Score 3 is equivalence. Score 4 is agree and Score 5 means strongly agree. We determine the acceptability and satisfaction at score 4 or 5. The score that lower than 4 is classified as poor acceptability. All the data of acceptability aspect were shown in table 2 . On the topic of easily to use, $85 \%$ of the subjects give the score 4 or 5 ; while, $15 \%$ of the subject give the score 3 or less. According to pain, $82 \%$ of the participants reported that they feel less pain and $18 \%$ gave score less than 4 . Reliability of the test is a factor that we considered. In this aspect, $37 \%$ of the participants gave score 3 or less and only $63 \%$ of them gave score 4 or 5 which were quite low comparing to other aspects of the questionnaire. Comfortable of the test usage, score 4 or 5 is $89 \%$ of the subjects. On embarrassment aspect, we ask the patient that comparing to do the Pap test by physicians; you feel this test is less embarrassment. Most of the participants $(80 \%)$ feel less embarrassment while using self-sampling device. The other factors of the acceptability questions are shown in the table 2

According to the price of the test, most of the participants will use this instrument if the price is less than 1,000 Baht. If the price is less than $1,000 \mathrm{Baht}, 82 \%$ of the subject will use it. If the price 1,000-2,000 Baht $17 \%$ and if the price is higher than 2,000 Bath, only $1 \%$ will use this instrument.

\section{Discussion}

Cervical cancer is considered as a major health problem in Thai women. Although Pap smears can reduce the incidence of cervical cancer, the number of new cervical cancer cases in Thailand is continuously increase because the coverage of Pap smears in Thailand is still low. The reason for poor coverage is varies. The coverage of the national policy is one of the causes. However, embarrassment and fear of vaginal examination are others important reasons for avoiding Pap smear in Thai women (Oranratanaphan et al., 2010).

From this study, the participants have ever done Pap smear is $81 \%$ which is higher than average of Thai women. There was a report about the coverage of Pap smear in Thai female. The result found that only $25-38 \%$ of Thai female aged 30-65 years have been screen at least once in their life time and the rest of them have never been screen (Sriamporn et al., 2006). Although, the percentage of participants that have ever done Pap smear in this study is high as $81 \%$, the participants that interval between the tests more than 1 year is more than $50 \%$. The reason of higher screening rate in this study because the subjects 
that attend this study are the patients at outpatient clinic that may not represent the whole Thai women in this aspect. However, the average income of the participants is the same as mean income of Thai people. Most of the participants of this study are also employee either full time or temporary which is the majority of the occupation in Bangkok people.

Obstacle of Pap smear in Thailand is not only the national policy, but also the embarrassment of Thai women (Sriamporn et al., 2006; Oranratanaphan et al., 2010). There was a study about the reason of avoid going for Pap smears. The major 4 reasons for avoiding Pap smear are embarrassment, fear of pain from speculum examination, no existing symptoms and too busy to go to a hospital. Self-sampling HPV testing is a new modality in Thailand for cervical cancer screening that women can screen the cancer by herself at home which can decrease the embarrassment and other reasons such as fear of speculum examination or too busy to go to the hospital for the test. However, the acceptability of self-sampling device is influenced by culture and behavior of people in each area. Thai women have many aspects of culture and behavior difference from European or American women. Those factors may influence the acceptability of the test. Therefore, the test of acceptability is important before introduce some innovation to the population.

According to the result of self-sampling HPV testing, the incidence of high risk HPV infection in this study is $16 \%$ which is higher than the global incidence (Bruni et al., 2010). The reason for higher incidence of high risk HPV infection may be due to the interval of screening. In Thailand, most of Pap test is conventional method and the interval between the tests should be 1 year. However, more than half of the participants in this study have interval between the tests more than 1 year.

From this study, the acceptability of self-sampling HPV testing is quite well but the reliability of the test is questioned by some participants. Acceptability of the selfsampling device in this study is good. The participants give score 4-5 which mean agree or highly agree in $80-90 \%$ in most of the aspect except the aspect of reliability of the test. Only $63 \%$ of the participants rely on this instrument. The problem of reliability is also considered in another study conducted by Berner A, et al (Berner et al., 2013). Form that study, the acceptability of the test is great but the patients still hesitate about the reliability of the test and the accuracy of collecting sample by themselves. Some patients did not sure that they can collect the specimen correctly. Those problems are the barrier of using selfsampling HPV testing confidently

Surprisingly, $20 \%$ of the participant in this study did not feel less embarrassment while using self-sampling device which contrasts to previous study. Previous studies in Western country usually revealed that there was less embarrassment in Self- sampling comparing to physician taken method (Dzuba et al., 2002; Arriba et al., 2010; Virtanen et al., 2011). The reason for the difference in the result may due to the cultural and behavioral differences. In Thailand, the instruments or medications that use vaginally are not as popular as in Western countries. Therefore, the embarrassment is affected by cultural and behavioral aspect in the population.

In the aspect of repeated use of this instrument, $79 \%$ of the participants may use this instrument in the next screening; while, $21 \%$ of the participants hesitate to use this instrument again. The price of the instrument is an important issue for making decision to use or not to use this instrument next time. For the country that the screening Pap smear is not totally covered by the Government like Thailand, some people have to pay for screening by themselves. Therefore, the cost of screening method may influence in acceptability of the test. From the result of this study, the participants respond that the test will be attractive if the cost is less than 1,000 Baht (32.59 Baht=1USD). If the cost is more than 1,000 Baht, this test will considered too expensive for them to pay (the average income of the participants of this study is 10,000-30,000 Baht per month). This aspect may different from other previous studies. The previous studies focused only on the acceptability not on the price because the Government in those studies pay for the test and send to the non- attendee to improve the coverage of the National screening policy which was different from the situation of screening in Thailand. Therefore, the price of the test may also influence the acceptability of the test in Thailand.

In conclusion, self-sampling HPV testing is a new modality for screening cervical cancer in Thailand. The acceptability of the test is good but some factors like cost of the test, confidence and reliability of the test may be obstacles for using self-sampling device in Thailand. This study is performed in a small group of outpatient participants that may not be implied to all Thai population in some aspects. However, this study is the first study that determines acceptability of self-sampling HPV DNA testing in the women in Thai culture. Asian women have different culture, believes and behavior from Western women. Therefore, the result in acceptability of the new modality used may be different. For the further implement, larger scales of participants especially in non-attendee group should be considered.

\section{References}

Anhang R, Nelson JA, Telerant R, Chiasson MA, Wright TC $\mathrm{Jr}$, (2005). Acceptability of self-collection of specimens for HPV DNA testing in an urban population. $J$ Womens Health (Larchmt), 14, 721-8.

Arriba LN, Enerson CL, Belinson S, Novick L, Belinson J, (2010). Mexican Cervical Cancer Screening Study II: acceptability of human papillomavirus self-sampler. Int $J$ Gynecol Cancer, 20, 1415-23.

Bais AG, van Kemenade FJ, Berkhof J, et al (2007). Human papillomavirus testing on self-sampled cervicovaginal brushes: an effective alternative to protect nonresponders in cervical screening programs. Int J Cancer, 120, 1505-10.

Berner A, Hassel SB, Tebeu PM, et al (2013). Human papillomavirus self-sampling in Cameroon: women's uncertainties over the reliability of the method are barriers to acceptance. J Low Genit Tract Dis, 17, 235-41.

Bruni L, Diaz M, Castellsagué X, Ferrer E, Bosch FX, de Sanjosé $S$, (2010). Cervical human papillomavirus prevalence in 5 continents: meta-analysis of 1 million women with normal cytological findings. J Infect Dis, 202, 1789-99.

Bundhamcharoen K, Teerawattananon Y, Vos T, et al, (2002). 
Burden of disease and injuries in Thailand, priority setting for policy. Nonthaburi: Bureau of Health Policy and Planning, Ministry of Public Health.

Dijkstra MG, Heideman DA, van Kemenade FJ, et al (2012). Brush-based self-sampling in combination with GP5+/6 \pm PCR-based hrHPV testing: high concordance with physician-taken cervical scrapes for HPV genotyping and detection of high-grade CIN. J Clin Virol, 54, 147-51.

Dzuba IG, Díaz EY, Allen B, et al (2002). The acceptability of self-collected samples for HPV testing $v s$. the Pap test as alternatives in cervical cancer screening. $J$ Womens Health Gend Based Med, 11, 265-75.

Ferlay J, Shin HR, Bray F, et al (2010). GLOBOCAN 2008:Cancer Incidence and Mortality Worldwide: IARC Cancer Base No. 10. Published 2010. http://globocan.iarc.fr.

Forrest S, McCaffery K, Waller J, et al (2004). Attitudes to self-sampling for HPV among Indian, Pakistani, AfricanCaribbean and white British women in Manchester, UK. $J$ Med Screen, 11, 85-8.

IARC monographs on the evaluation of carcinogenic risks to humans. Lyon: International Agency for Research on Cancer, 2005

Kececiglu M, Seckin B, Baser E, et al (2013). Cost and effectiveness comparison of immediate colposcopy versus human papillomavirus DNA testing in management of aypical squamous cells of undermined significance in Turkish women. Asian Pac J Cancer Prev, 14, 511-4.

Lindell M, Sanner K, Wikström I, Wilander E (2012). Self-sampling of vaginal fluid and high-risk human papillomavirus testing in women aged 50 years or older not attending Papanicolaou smear screening. BJOG, 119, 245-8.

Ministry of Public Health (2008). The Survey Report of Behavioural Risk Factors of Noncommunicable Diseases and Injuries in Thailand, 2005. Nonthaburi: MOPH; 2008.

Ngan HY, Garland SM, Bhatla N, et al (2011). Asia oceania guidelines for the implementation of programs for cervical cancer prevention and control. J Cancer Epidemiol, 2011, 794861.

Oranratanaphan S, Amatyakul P, Iramaneerat K, Srithipayawan S (2010). Knowledge, attitudes and practices about the Pap smear among medical workers in Naresuan University Hospital, Thailand. Asian Pac J Cancer Prev, 11, 1727-30.

Pandey S, Mishra L, Chandrawati (2012). Human papillomavirus screening in North India women. Asian Pac J Cancer Prev, 13, 2643-6.

Sahin MK, Sahim G, Dikici MF, Igde FA, Yaris F (2014). Women's perceptions and atitudes about cervical cancer in Turkey: Kato's device as an alternative to Pap smear. Asian Pac J Cancer Prev, 15, 905-10.

Schiffman M, Kjaer SK (2003). Natural history of anogenital human papillomavirus infection and neoplasia. J Natl Cancer Inst Monogr, 31, 14-9.

Snijders PJ, Verhoef VM, Arbyn M, et al (2013). High-risk HPV testing on self-sampled versus clinician-collected specimens: A review on the clinical accuracy and impact on population attendance in cervical cancer screening. Int $J$ Cancer, 132, 2223-36.

Sriamporn S, Khuhaprema T, Parkin M (2006). Cervical cancer screening in Thailand: an overview. J Med Screen, 13, 39-43.

Stenvall H, Wikström I, Backlund I, Wilander E (2007). Accuracy of HPV testing of vaginal smear obtained with a novel self-sampling device. Acta Obstet Gynecol Scand, 86, 16-21.

Szarewski A, Cadman L, Ashdown-Barr L, Waller J(2009). Exploring the acceptability of two self-sampling devices for human papillomavirus testing in the cervical screening context: a qualitative study of Muslim women in London. $J$
Med Screen, 16, 193-8.

Szarewski A, Cadman L, Mallett S (2007). Human papillomavirus testing by self-sampling: assessment of accuracy in an unsupervised clinical setting. J Med Screen, 14, 34-42.

Tamalet C, Le Retraite L, Leandri FX, et al (2013). Vaginal self-sampling is an adequate means of screening HR-HPV types in women not participating in regular cervical cancer screening. Clin Microbiol Infect, 19, 44-50.

Thurston WE, Scott CM (2005). Barriers to screening: A critical review of the literature (1990-1995). Ottawa, ON: Health Canada.

van Baars R, Bosgraaf RP, ter Harmsel BW, et al (2012). Dry storage and transport of a cervicovaginal self-sample by use of the Evalyn Brush, providing reliable human papillomavirus detection combined with comfort for women. J Clin Microbiol, 50, 3937-43.

Virtanen A, Anttila A, Luostarinen T, Nieminen P (2011). Selfsampling versus reminder letter: effects on cervical cancer screening attendance and coverage in Finland. Int J Cancer, 128, 2681-7.

Virtanen A, Nieminen P, Luostarinen T, Anttila A (2011). Selfsample HPV tests as an intervention for nonattendees of cervical cancer screening in Finland: a randomized trial. Cancer Epidemiol Biomarkers Prev, 20, 1960-9.

WHO (2006). Comprehensive Cervical Cancer Control: A Guide to Essential Practice. Geneva, Switzerland: World Health Organization.

Working group of Burden of Disease Project. Burden of disease and injuries in Thailand, 2004 (interim report). Nonthaburi: International Health Policy Program, Ministry of Public Health, 2007.

Zhang L, Lin Y, Li JK (2014). Concordance in cervical HPV detection between Hybrid Capture 2 and HPV GenoArray tests. Asian Pac J Cancer Prev, 15, 4465-6. 